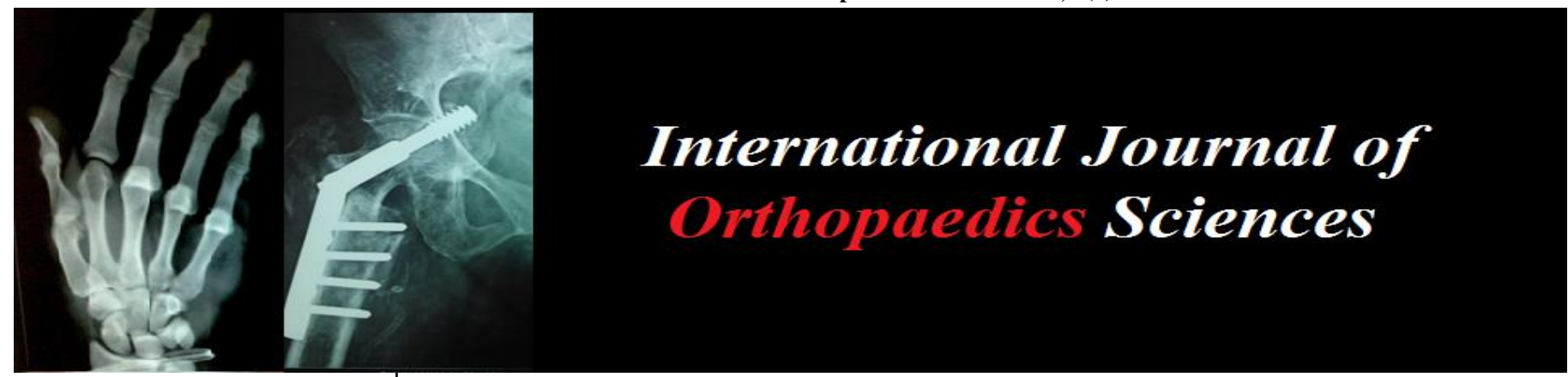

ISSN: $2395-1958$

IJOS 2018; 4(3): 298-304

(C) 2018 IJOS

www.orthopaper.com

Received: 24-05-2018

Accepted: 25-06-2018

Dr. Ullas Mahesh

Assistant Professor,

Department of Orthopaedics,

Bangalore, Karnataka, India

Dr. Anil Patil

Assistant Professor,

Department of Orthopaedics,

Bangalore, Karnataka, India

Correspondence

Dr. Anil Patil

Assistant Professor,

Department of Orthopaedics,

Bangalore, Karnataka, India

\section{Medial patellofemoral ligament reconstruction using dual patella docking technique}

\author{
Dr. Ullas Mahesh and Dr. Anil Patil
}

DOI: https://doi.org/10.22271/ortho.2018.v4.i3f.54

\section{Abstract}

Introduction: Medial patellofemoral ligament (MPFL) injuries are common in children and young adults.

Patellar dislocation is the complete displacement of the patella from the trochlear groove of the femur. It nearly always involves a lateral displacement and commonly occurs during sports.

Current techniques of medial patellofemoral ligament (MPFL) reconstruction vary with respect to methods of fixation on the femur and the patella. In patients with recurrent patellar dislocations with normal lower-extremity alignment, anatomic reconstruction of the MPFL has been shown to restore patellar stability. We describe a technique that creates an anatomic reconstruction using a dual docking technique into the patella. Our technique is simple and efficacious for reconstructing the MPFL without implant fixation in the patella, allowing a maximal bone-tendon interface for healing.

Results: It provided anatomic reconstruction, not reliant on implants in patella, Docking technique allows greater surface area for graft-to-bone contact.

Discussion: The orthopedic literature is replete with techniques for the management of patella instability. These techniques include proximal soft-tissue procedures, distal osseous realignment procedures, and combinations of both. We are using Dual Patella docking technique here.

Keywords: Medial patellofemoral ligament (MPFL) reconstruction; patellar dislocation; patellar subluxation, docking

\section{Introduction}

The medial patellofemoral ligament (MPFL) has been shown to be the primary stabilizing softtissue structure of the patellofemoral joint. Several studies, both anatomic and biomechanical, have shown that the MPFL is the most important restraint to lateral patellar translation from 0 to 30_ of flexion. Because of its importance, various techniques have been described to reconstruct the MPFL in chronic lateral patellar instability. The soft-tissue elements that contribute to patellofemoral stability include the medial patellofemoral ligament (MPFL), vastus medialis obliquus (VMO), medial retinaculum, and the medial patellomeniscal and patellotibial ligaments. Biomechanical studies demonstrate that the MPFL accounts for $50 \%$ to $60 \%$ of the medial soft-tissue restraining force resisting lateral patellar subluxation or dislocation. Because of its critical role in stabilizing the patella, several MPFL reconstruction procedures have been developed.

\section{Materials \& Methods Eligibility Criteria}

A one year prospective study was done with 20 patients, with a minimum 12-month follow-up, were included in Bangalore. A minimum follow-up of 12 months was chosen based on the time that patients get back to a normal level of function and sporting activities. all patients that had a first-time patellar dislocation, continued patellar instability, patellar subluxation, or recurrent patellar dislocation who underwent MPFL reconstruction using dual patellar docking technique with graft were included. Patients who had previous knee operations were also eligible, as this is the common clinical scenario. Subjects that underwent lateral release at the time of surgery were not excluded. However, those having any additional stabilization procedure at the time of MPFL reconstruction were excluded from the study. Studies that focused on MPFL repair were also excluded. 


\section{Diagnosis and preoperative planning}

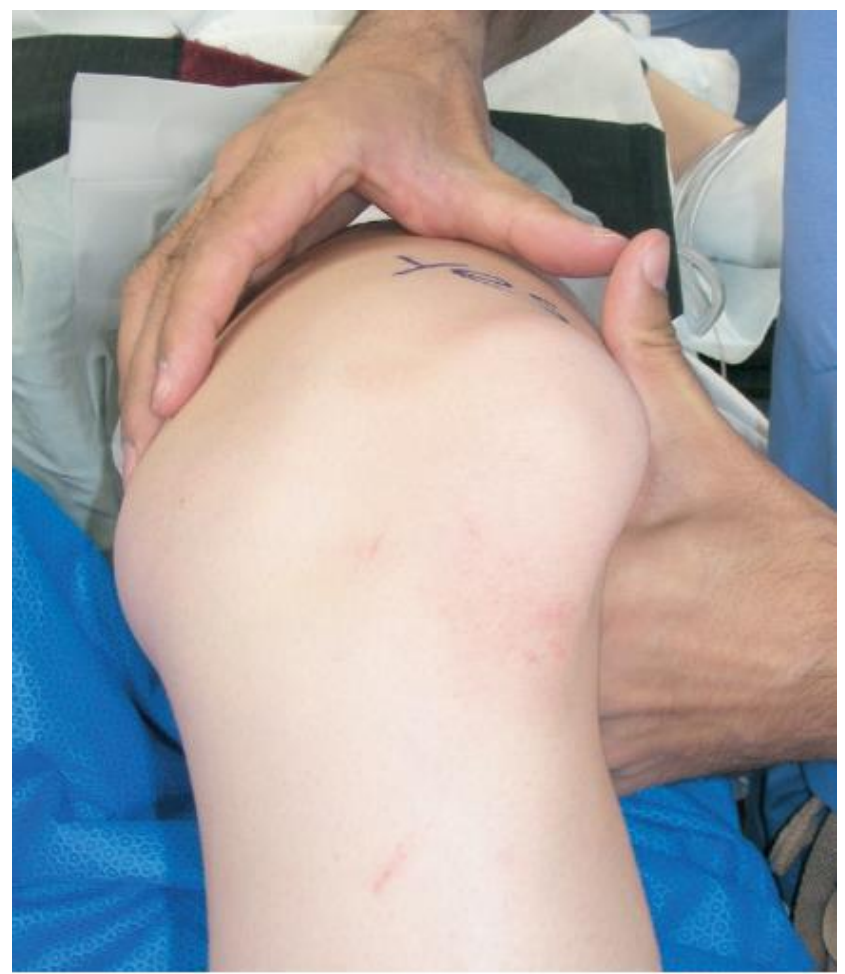

Eliciting Patellar dislocation laterally

Examination should include evaluation for an effusion, which may indicate intra-articular pathology.

Tenderness over the medial epicondyle can indicate an injury to the MPFL. Patellar mobility is assessed in full extension and compared with the contralateral side, with patellar apprehension and lateral patellar quadrant translation with a firm versus soft endpoint. Patellar tracking is assessed by evaluating for the $\mathbf{J}$ sign.

Limb alignment is assessed for the presence of femoral anteversion, genu valgum, and external tibial torsion.

Hip and quadriceps muscular strength should be assessed. Generalized ligamentous laxity is assessed with evaluation for the presence of elbow and knee recurvatum, metacarpal hyperextension, passive apposition of the thumb to the volar forearm, and active forward flexion of the trunk with the knees fully extended so that the palms of the hands rest flat on the floor. Radiographic evaluation should include standard anteroposterior, lateral, and axial weight-bearing views. Careful evaluation for intra-articular bodies and osteochondral fractures should be performed. The lateral radiograph can be used to assess for patellar height and depth of the femoral trochlea. The axial radiograph can be used to assess the femoral sulcus angle, congruence angle, lateral patellofemoral tilt angle, lateral shift ratio, and absolute lateral patellar displacement.

Computed tomography is used to measure the tibial tubercle and trochlear groove distance. Magnetic resonance imaging can be used to evaluate other injuries after a patellar dislocation, including a bone contusion on the lateral femoral condyle and medial patella, as well as an MPFL tear and articular cartilage injury.

\section{Indications and contraindications}

There is debate in the literature concerning when surgical intervention is indicated for patellar instability.

Our indications include recurrent instability despite a trial of nonoperative treatment that typically includes patella- stabilizing braces and physical therapy to include quadriceps, hip, and core muscle strengthening. In patients with osteochondral injuries and intra-articular bodies, arthroscopy is indicated for the treatment of these injuries and concomitant MPFL reconstruction. Contraindications to an isolated MPFL reconstruction include limb rotation and alignment abnormalities.

\section{Technique}

The patient is placed supine on the table. An intraoperative examination is performed to confirm clinical findings. We perform a single-portal diagnostic arthroscopy from the superolateral portal of the knee, allowing us to evaluate the cartilage, patellofemoral tracking, and laxity of the medial joint restraining ligaments.

Arthroscopic treatment of any noted abnormalities is performed as necessary through standard portals.

\section{Graft Preparation}

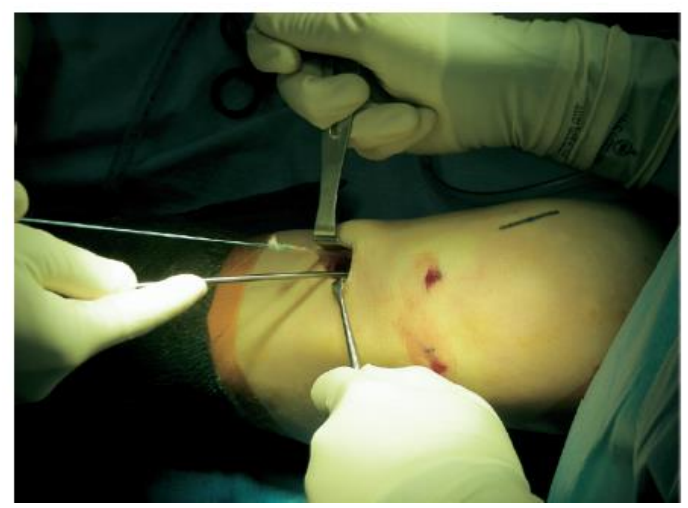

Harvesting of the semitendinosus tendon with a tendon stripper.

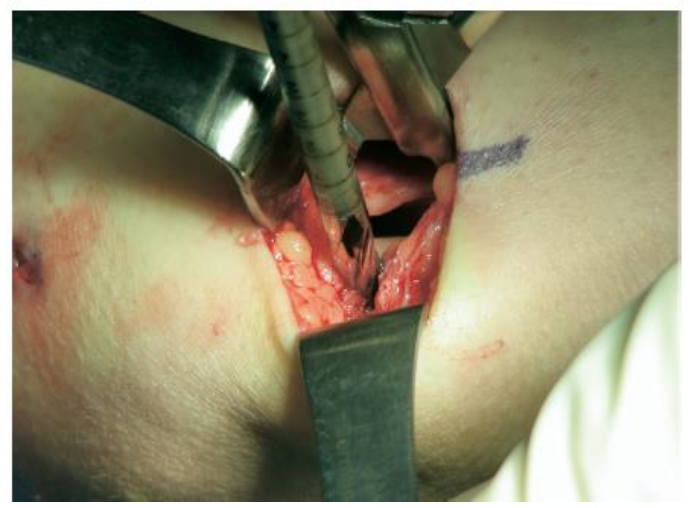

Drilling the patella docking over the guide pin.

We use a semitendinosus allograft doubled over. The folded end is sutured together with a locking stitch with the free ends exiting the end of the graft. Two Fiber- Wire sutures (Arthrex, Naples, FL) are run through the 2 graft limbs individually. The diameters of the folded end and 2 graft limbs are measured and used to size the femoral tunnel and patellar tunnels, respectively. The graft is placed on a tensioning device and wrapped in a moist sponge. An example of our graft after preparation is shown in Femoral Tunnel.

We use a C-arm to obtain a perfect lateral view of the knee. The MPFL insertion site has been shown radiographically to be just anterior to the distal osterior cortical line and superior to the Blumensaat line. We place a Beath pin against the skin and confirm the location of the tip. Care should be taken during this step in skeletally immature patients because the 
femoral attachment of the MPFL is near the distal femoral physis and may need to be adjusted if a physeal-sparing approach is indicated. A skin incision is made, and the Beath pin, starting at the correct insertion site, is advanced across the femur parallel to the knee joint and out the lateral skin surface. We aim slightly proximally and anteriorly to avoid the risk of violating the intercondylar notch and trochlea, as well as to avoid damage to the neurovascular structures. If more than 2 cortices are encountered during passing of the pin, then fluoroscopy can be used to determine whether the femoral trochlea or notch has been violated and the pin should be redirected. A cannulated reamer, corresponding to the measured diameter of the folded graft end, is used to drill over the pin to a depth of $25 \mathrm{~mm}$. Multiple structures attach near the femoral attachment of the MPFL, including the superficial medial collateral ligament, posterior oblique ligament, medial head of the gastrocnemius, and adductor magnus tendon.29 To our knowledge, the clinical significance of potentially injuring these structures while reaming has not been elucidated. A looped suture is then shuttled through the femur using the Beath pin, pulling the pin out laterally.

The looped end is left medial to shuttle the sutures from the folded graft end out the femur. The graft is tensioned into the femoral tunnel. A guide pin is placed into the tunnel, and an interference screw (Arthrex) is used to secure the graft into the femoral tunnel; the surgeon should then ensure that the graft is securely fixed.

\section{Materials \& methods}

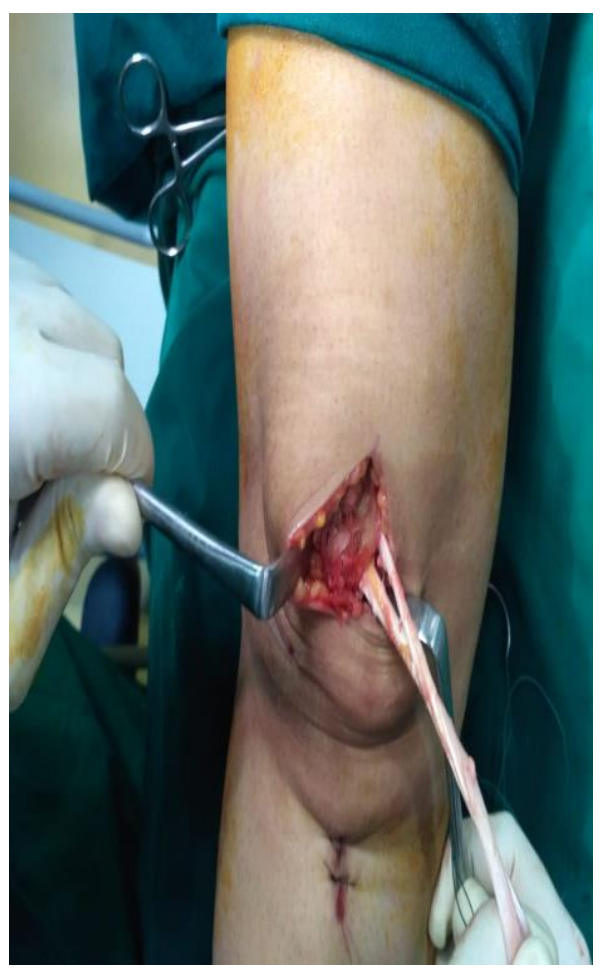

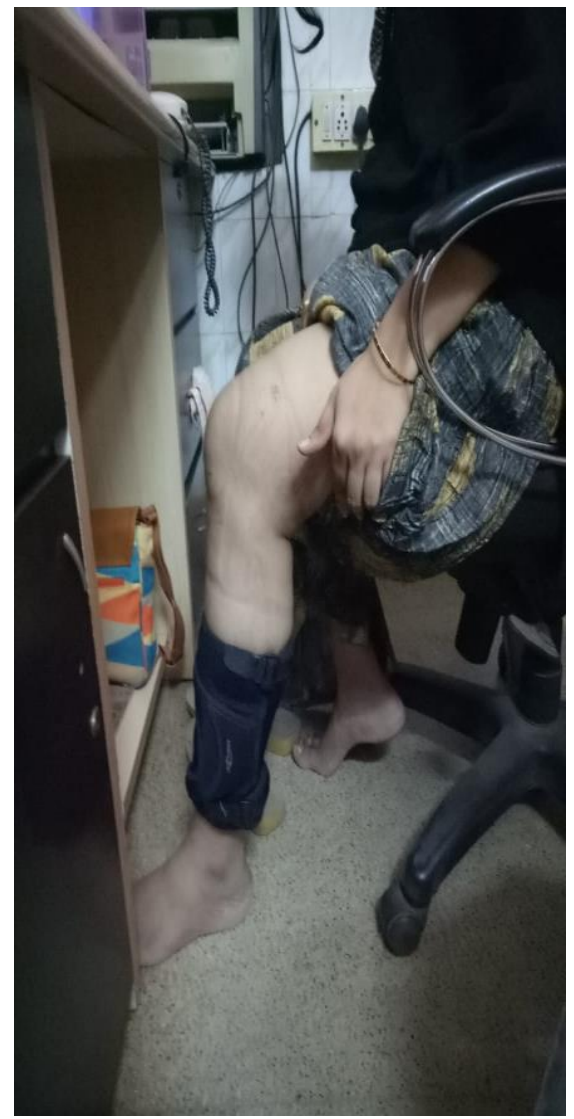

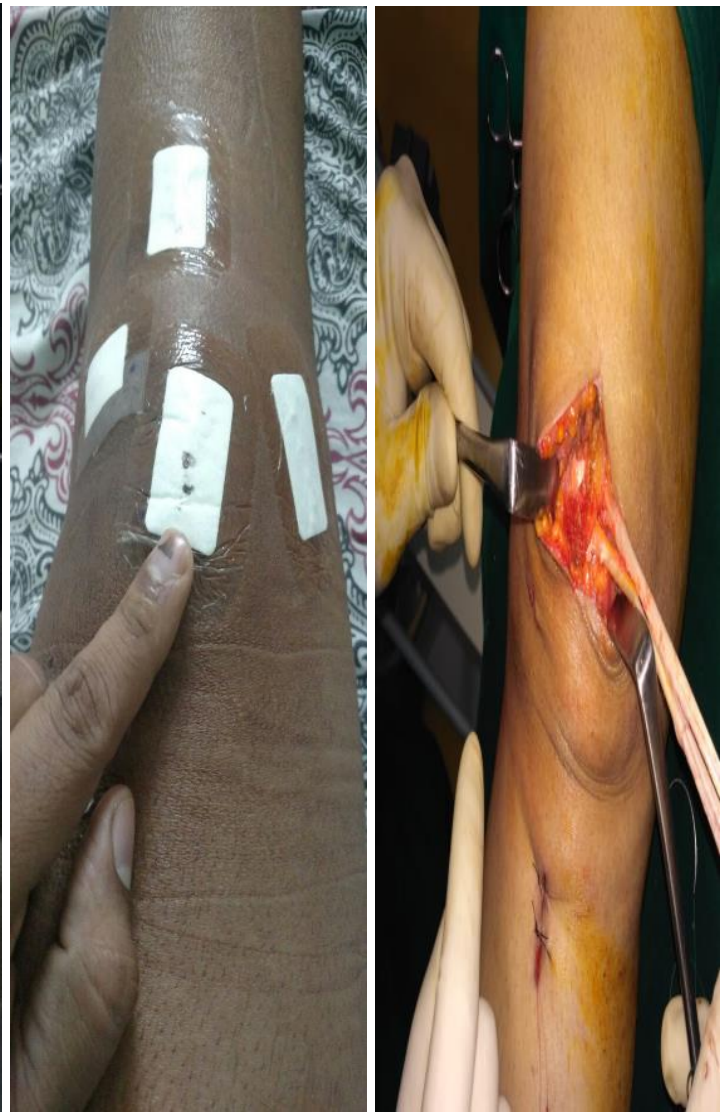




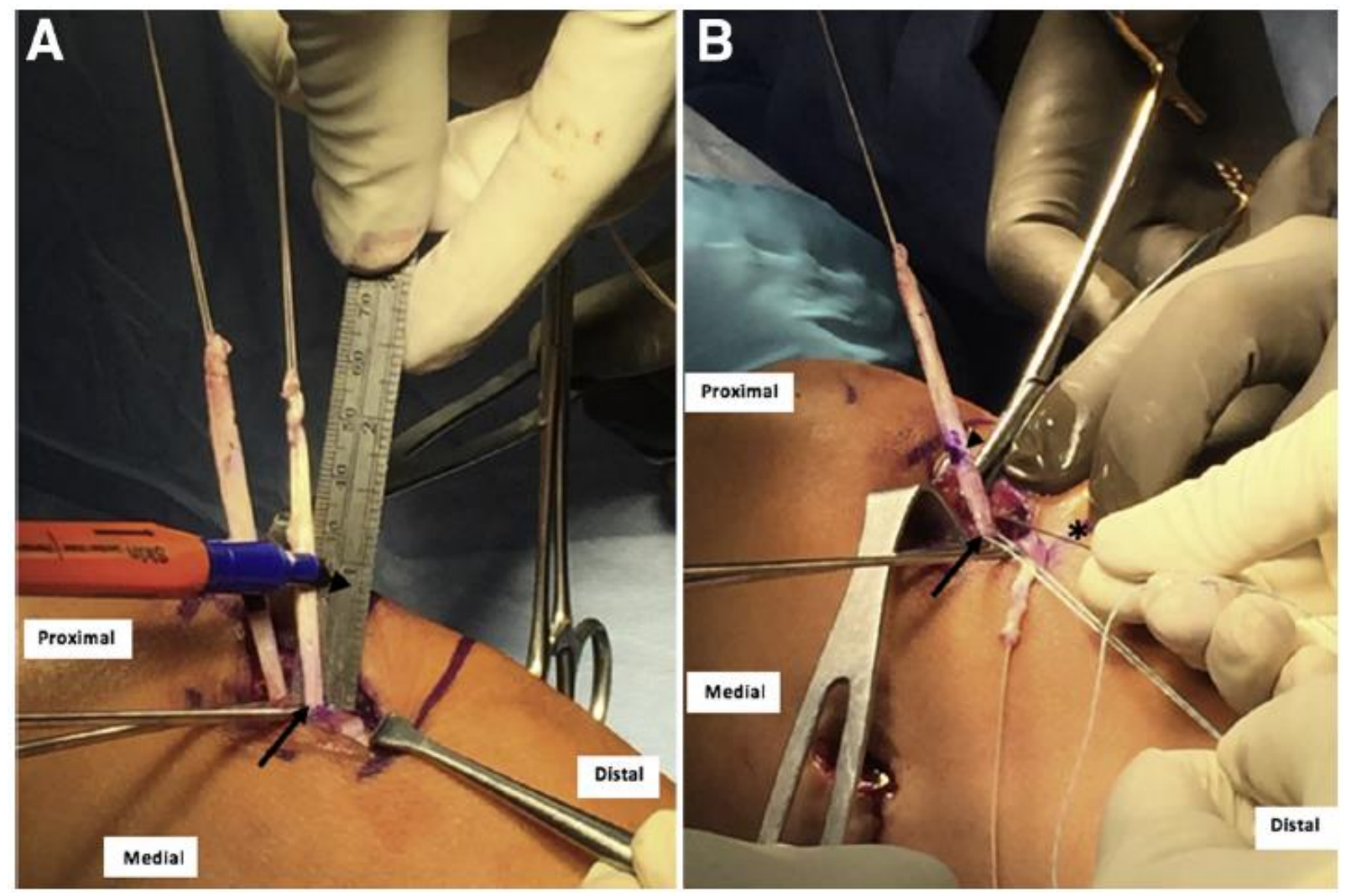

Photographs of a left knee in a patient positioned supine with proximal to the left and distal to the right. (A) Measurement of graft limbs. We mark the graft $2 \mathrm{~cm}$ distal (arrowhead) to the point at which it makes contact with the medial cortex of the patella (arrow). (B) Suturing of grafts with straight needle (asterisk) and a looped fiber wire suture form the point of contact with the medial cortex of the patella (arrow) to the marked point $2 \mathrm{~cm}$ distal (arrowhead.).

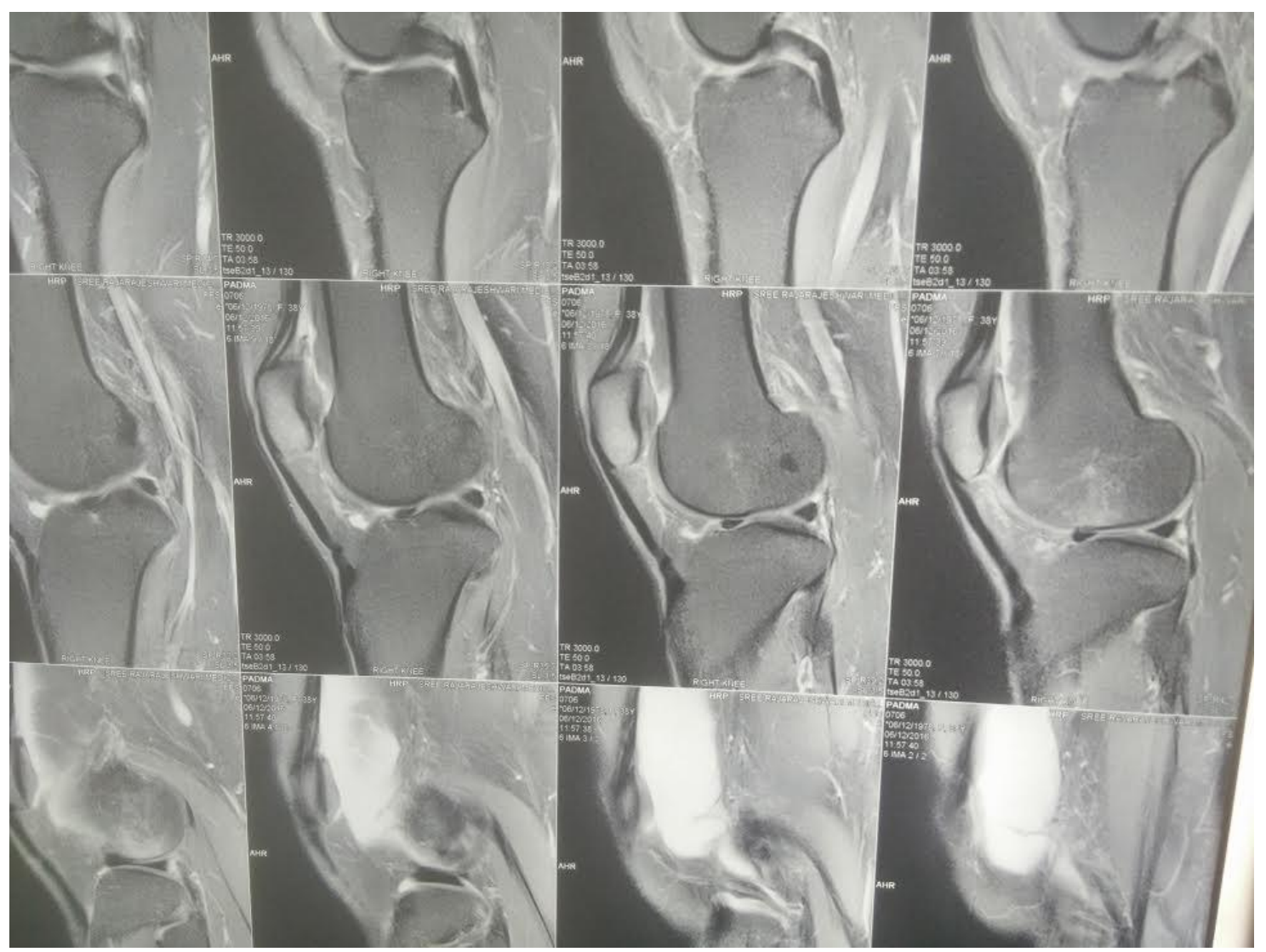


International Journal of Orthopaedics Sciences
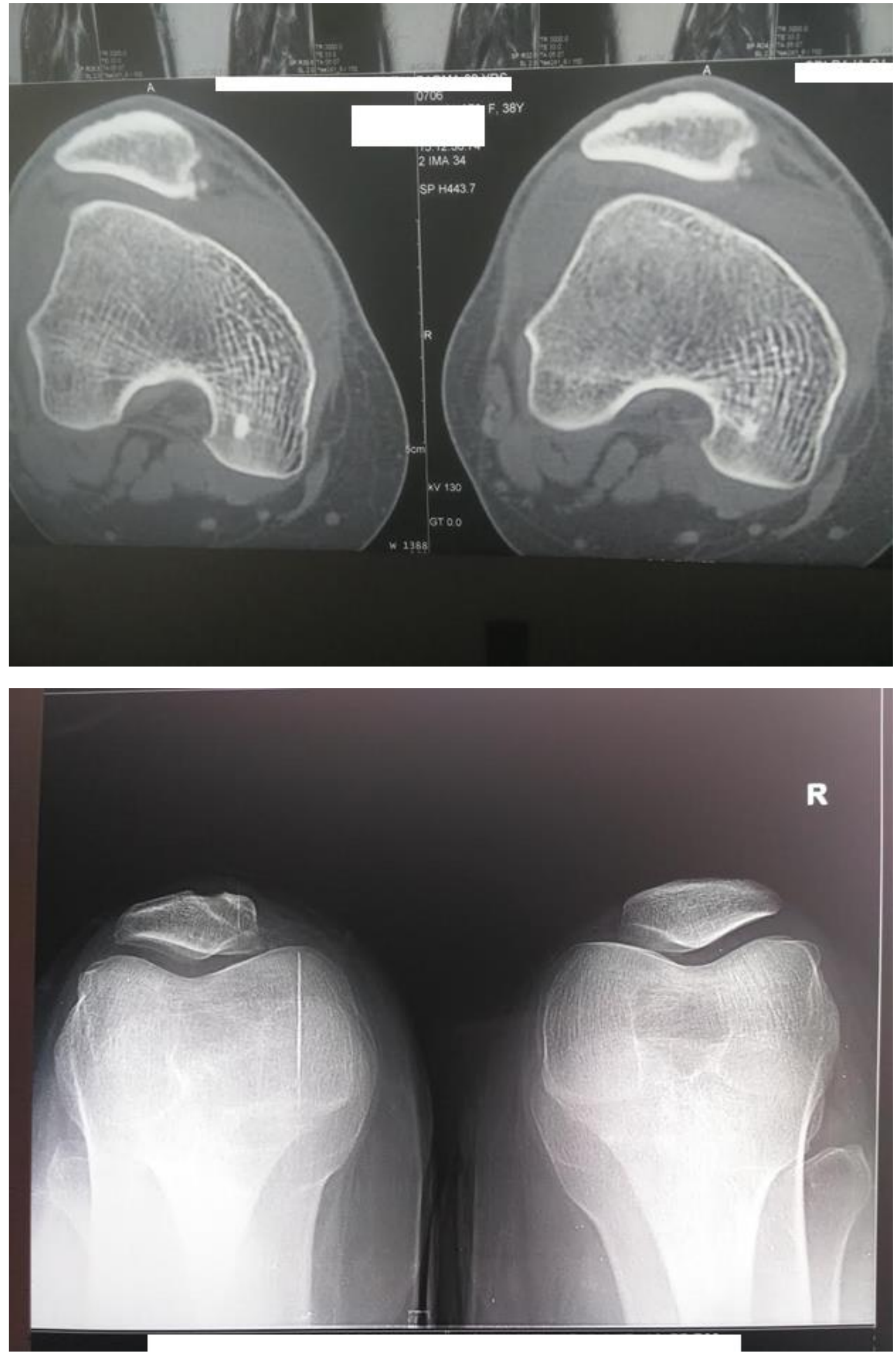


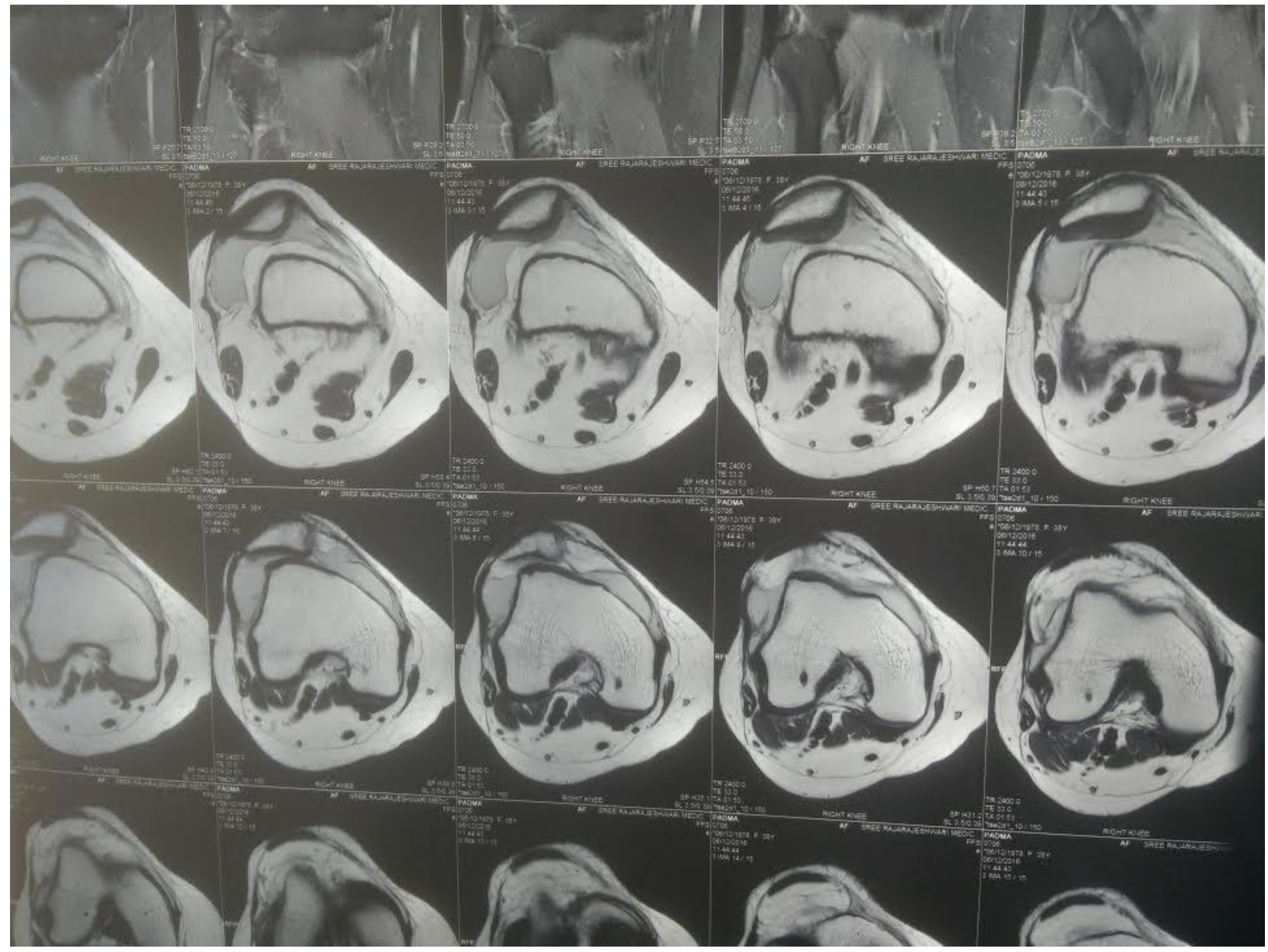

\section{Discussion}

A one year prospective study was done with 20 patients, with a minimum 12-month follow-up, were included in Bangalore. The MPFL is a distinct structure located between the second and third layers of the medial joint capsule, originating in a depression between the medial epicondyle and the adductor tubercle. Its insertion on the patella is broad, attaching along the proximal twothirds in a fan-shaped manner. Kang et al. described 2 functional bundles of the MPFL. Use of a 2-limb graft technique, as we describe, as opposed to single graft limb patellar insertion allows for more accurate re-creation of these bundles and insertion on the MPFL. Mechanical studies have shown the importance of the MPFL in its contribution to the restraining force against lateral displacement of the patella, contributing $53 \%$ to $60 \%$ of restraining force against lateral patellar translation. The importance of this ligament in chronic lateral patellar instability in a patient with normal osseous anatomy and limb alignment has led to the development of various surgical techniques to reconstruct the MPFL with variations in graft choice, anatomic versus nonanatomic reconstruction, and fixation methods. Several studies have shown the importance of an anatomic reconstruction of the femoral attachment. A femoral tunnel that is too proximal may lead to graft laxity in extension and over-tensioning in flexion, a femoral tunnel that is too distal may lead to graft over-tensioning in extension and laxity in flexion, and tunnel placement too anterior can cause overloading of the medial patellofemoral cartilage. The fixation method to the patella can be generalized as tunnel versus suture fixation. A systematic review showed a higher risk of recurrent dislocation or subluxation (4.8\% vs $3.3 \%$ ) and hypermobility or apprehension (24\% vs $8.6 \%)$ with suture fixation. However, a trend toward a higher overall complication rate was noted with the tunnel technique $(30 \%$ vs $22 \%$ ).

We describe a technique that re-creates an anatomic reconstruction of the MPFL at both the femoral and patellar attachments. Our method of fixation, using a dual docking technique, has potential benefits It creates 2 incomplete transverse tunnels that do not rely on implants for fixation. The docking technique is well described for ulnar collateral ligament reconstruction of the elbow and has been shown to have favorable outcomes. Our use of this technique allows for increased surface area for graft-to-bone healing. Our dual docking technique also simplifies tunnel placement by obviating the need to converge tunnels, potentially decreasing surgical time and decreasing risk of fracture. A single-tunnel MPFL docking technique has been described and shown to have good outcomes.

Potential limitations of our described method include dualtunnel placement, which may lead to increased risk of fracture compared with single-tunnel or suture anchor fixation. Improper angling and positioning of the Beath pin may cause inadvertent iatrogenic chondral damage if it penetrates the joint.

\section{Advantages}

- Anatomic reconstruction was obtained

- This technique is not reliant on implants in patella

- Dual patellar docking technique allows greater surface area for graft to bone contact

- Parallel tunnels in patella simplify previously described technique.

\section{Disadvantages}

- Dual tunnels may lead to increased risk of fracture in the patella 
- Tensioning of graft can be difficult with docking technique

\section{Conclusion}

In conclusion, we did a study on 20 patients, describe a surgical technique with potential benefits creating an anatomic reconstruction of the MPFL using 2 patellar tunnels to secure each graft limb. We believe that a more anatomic recreation of the MPFL broad insertion on the patella may be of benefit, especially in the very active patient. In addition, docking the grafts individually allows for the use of 2 smaller tunnels as opposed to 1 larger tunnel. Other authors have described 4-tunnel fixation in the patella. The benefit of our technique is the ability to accurately restore the anatomy while limiting the chance of fracture caused by 4 tunnels. So we conclude that Medial Patellofemoral Ligament Reconstruction Using Dual Patella Docking Technique, is much better than a single tunnel or 4tunnel technique.

\section{References}

1. Miswan MFBM, Al-Fayyadh MZM, Seow Hui T, Mohamed Ali MRB, Ng WM. Soft-tissue loop for medial patellofemoral ligament reconstruction. Arthrosc Tech. 2016; 5:e321-e327.

2. Brief LP, Laico JP. The superolateral approach: A better view of the medial patellar plica. Arthroscopy. 1987; $3: 170$.

3. http://ovidsp.ovid.com/ovidweb.cgi?T1/4JS\&PAGE $1 / 4$ reference\&D1/4med2\&NEWS1/4N\&AN1/43675788.

4. Johnson D. Anterior cruciate reconstruction with hamstrings and bioscrews.

http://www.carletonsportsmed.

com/anterior_cruciate_reconstruction.htm. Accessed May 20, 2017.

5. Herschel R, Hasler A, Tscholl PM, Fucentese SF. Visualpalpatory versus fluoroscopic intraoperative determination of the femoral entry point in medial patellofemoral ligament reconstruction. Knee Surg Sports Traumatol Arthrosc. 2017; 25:2545-2549.

6. Ahmad CS, Brown GD, Stein BS. The docking technique for medial ligament reconstruction: surgical technique and clinical outcome. Am J Sports Med. 2009; 37:20212027.

7. Balcarek P, Walde TA. Accuracy of femoral tunnel placement in medial patellofemoral ligament reconstruction: The effect of a nearly true-lateral fluoroscopic view. Am J Sport Med. 2015; 43:2228-2232.

8. Schöttle PB, Schmeling A, Rosenstiel N, Weiler A. Radiographic landmarks for femoral tunnel placement in medial patellofemoral ligament reconstruction. Am J Sports Med. 2007; 35:801-804.

9. Nguyen CV, Farrow LD, Liu RW, Gilmore A. Safe drilling paths in the distal femoral epiphysis for pediatric medial patellofemoral ligament reconstruction. Am J Sports Med. 2016, 363546516677795.

10. Shea KG, Styhl AC, Jacobs JC. The relationship of the femoral physis and the medial patellofemoral ligament in children: A cadaveric study. Am J Sports Med. 2016; 44:2833-2837.

11. Farrow LD, Alentado VJ, Abdulnabi Z, Gilmore A, Liu RW. The relationship of the medial patellofemoral Dual Patella Docking Technique e2099 ligament attachment to the distal femoral physis. Am J Sports Med. 2014; 42:2214-2218

12. Stefancin J, Parker R. First-time traumatic patellar dislocation: a systematic review. Clin Orthop Relat Res. 2007; 455:93-101.

13. Steiner TM, Torga-Spak R, Teitge RA. Medial patellofemoral ligament reconstruction in patients with lateral patellar instability and trochlear dysplasia. Am J Sports Med. 2006; 34:1254-1261. 